\section{Clinical Experience of Intensity Modulated Radiotherapy Pre-Treatment Quality Assurance for Carcinoma Head and Neck Patients with EPID and IMatriXX in Rural Center}

\author{
More M. ${ }^{1 * \odot}$, Jain V.2 , Gurjar O.P. ${ }^{3}$
}

\begin{abstract}
Background: Radiation therapy techniques as Intensity Modulated Radiotherapy (IMRT), rapid arc have been used for treatment of cancer with high accuracy.

Objective: Verification of planned and delivered dose distribution is important, therefore current study aims to analyse quality assurance (QA) results of IMRT by Electronic Portal Imaging Device (EPID) and IMatriXX in head and neck Carcinoma (Ca H\&N) patients.
\end{abstract}

Material and Methods: In this experimental study, performance of an EPID and IMatriXX was assessed with dose measurements using ionization chamber. Calibrated IMatriXX and EPID are used for pre-treatment patient specific quality assurance (PSQA), for 122 patients' plans of $\mathrm{Ca} H \& N$ with IMRT treatment technique on linear accelerator. Dose images were acquired and compared with gamma evaluation $(3 \% / 3 \mathrm{~mm})$ and three scalar parameters, named average $\gamma\left(\gamma_{\text {avg }}\right)$, maximum $\gamma\left(\gamma_{\max }\right)$ and area gamma $<1$, were analyzed in the region of interest.

Results: The $\gamma$ correlation comparisons yielded average correlation of 0.990 and 0.982 for IMatriXX and EPID respectively. Maximum value of gamma is 0.998, while minimum gamma is 0.872 for IMatriXX and 0.953 for EPID. For students, unpaired ' $\mathrm{t}$ ' test analysis is applied for comparison to two data sets. P-value was set at 0.005 which, for this study, was computed 0.001 , showing good correlation between measured data with IMatriXX and EPID.

Conclusion: The EPID and IMatriXX have significantly improved dosimetric properties, resulting in more sensitive, accurate measurements before actual treatment. The result shows EPID can be replaced with other dosimetry method and ionization chamber measurements. Portal imager is an efficient, accurate and sensitive dosimetry tool and is also the basis of pre-treatment quality assurance protocol.

Citation: More M, Jain V, Gurjar OP. Clinical Experience of Intensity Modulated Radiotherapy Pre-Treatment Quality AssurCitation: More M, Jain V, Gurjar OP. Clinical Experience of Intensity Modulated Radiotherapy Pre-Treatment Quality Assur-
ance for Carcinoma Head and Neck Patients with EPID and IMatriXX in Rural Center. J Biomed Phys Eng. 2020;10(6):691-698. doi: 10.31661/jbpe.v0i0.2004-1102

\section{Keywords}

Radiotherapy; Intensity-Modulation; Calibration; Dosimetry; Quality Control

\section{Introduction}

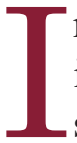
n Radiation therapy, some new techniques are evolved like IMRT, image guided radiotherapy (IGRT). These techniques require extensive and accurate dose verification methods that one of such method is the use of Electronic Portal Imaging Device (EPID). This method uses
${ }^{1}$ PhD Candidate, Department of Radiotherapy

and Oncology, Rural

Medical College, Pravara

Institute of Medical Sci-

ences (PIMS), Loni, India

${ }^{2} \mathrm{MD}$, Department of

Radiotherapy and

Oncology, Rural Medical

College, Pravara Insti-

tute of Medical Sciences

(PIMS), Loni, India

${ }^{3} \mathrm{PhD}$, Government Can-

cer Hospital, Mahatma

Gandhi Memorial Medi-

cal College, Indore, India

*Corresponding author:

M. More

Department of Radio-

therapy and Oncology,

Rural Medical College,

Pravara Institute of Med-

ical Sciences (PIMS),

Loni, India

E-mail: mahendra.

moremv@gmail.com

Received: 14 April 2020

Accepted: 21 June 2020 
the portal imager mounted on LINAC, which acquires images for dose verification and according to the studies, these portal images contain information related to dosimetry.

The dose-response characteristics have been described in prior studies, that portal images are useful for dosimetric verification [1-6]. The results of these studies show that the pixel signal given by portal imager is linear with dose. This signal may be converted to absolute dose. This requires the measurement of response over the range of various parameters. The response of the EPID varies by about $\pm 0.5 \%$ with time if there is no failure in electronic components [7].

Intensity modulated radiotherapy plans are very complex and dose distribution needs dosimetry verification using tools in two dimensions. Earlier dosimetry films were used for dose verification. EPID and IMatriXX have the advantages over films for dose verification in IMRT plan. Performing EPID and IMatriXX dose measurement is simple, requiring very less set-up time. These measurements can be done in a repetitive way and data is obtained in digital format very quickly, but dosimetry films need more time for processing and digitizing data. Also, for dosimetry films, the film batch requires to generate a calibration curve before its use for dosimetry.

Recording and storage of PSQA measurements are organized effectively when images are available in digital form. Radiation therapy departments require medical data to be available in digitized format, hence film dosimetry has become more scant and thus alternative devices are needed for radiation therapy dosimetry [8].

The EPID is mounted to the LINAC gantry and has advantage of easy setup with greater resolution. Thus, it does not require additional hardware for imaging and dosimetry and can also be used in various ways to verify a treatment plan before its actual delivery. The linear accelerator (LINAC), Varian Clinac DBX (Varian Medical Systems Palo Alto, USA) with $6 \mathrm{MV}$ photon energy was used in this study. EPID and IMatriXX were calibrated using 0.6 cc ionization chamber [9].

Earlier studies showed that the detector can be placed inside a phantom and measurements can be done [10], but EPID dose images can be measured at the detector plane. These portal dose images (PDI) are used to generate the dose in a plane or within the patient volume or in a phantom. Studies have reported the possibility of PDI, used in verification of treatment fields in IMRT [11-14].

The commissioning of LINAC and treatment planning system (TPS) - Eclipse version 11.0 for clinical use with a new inverse treatment planning method IMRT for H\&N cancer patient were clinically implemented in this rural cancer center. Most of advanced 3D dosimetry equipment was not available to verify the delivered dose in IMRT plan with accurate and efficient means. The study was designed to demonstrate and evaluate how IMatriXX and EPID dosimetry system can be used effectively for routine pretreatment PSQA in H\&N cancer patients which is demand of the clinic. Further to study, the sensitivity of gamma criteria and results of study can be used to set the baseline values for routine pretreatment IMRT plan verification.

\section{Material and Methods}

\section{Patient plans}

In this experimental study, IMRT treatment plans were analyzed for the $122 \mathrm{H} \& \mathrm{~N}$ cancer patients planned with treatment planning system (TPS) - Eclipse version 11.0 (VMS, Palo Alto, USA). For all 122 patients, treatment plan was generated with a five, seven or nine field dynamic IMRT technique with prescription given to various target volumes like Gross Tumor Volume (GTV), PTV and subsequently treated for $\mathrm{H} \& \mathrm{~N}$ cancer cases.

The treatment plans were generated with various optimizing methods to achieve prescribed dose to PTV and better sparing of Or- 
Pre-treatment Quality Assurance with EPID

gans at Risk (OARs) such as parotids, spine, oral cavity etc. IMRT plan with seven to nine fields was generated with appropriate OAR and target priorities. The dose grid of 0.25 $\mathrm{cm}^{3}$ resolution is used for plan optimization. The maximum dose, prescribed to the PTV is 74 Gy, which is delivered in 37 fractions and IMRT treatment plan were delivered with 6 MV energy photon beams.

\section{EPID \& IMatriXX dosimetry}

Verification plans are generated for each treatment using EPID a-Si 1000 (VMS, Palo Alto, USA) and IMatriXX ion chamber array device (IBA Dosimetry GmbH, Germany). The gantry angle was set to $0^{\circ}$ for both IMatriXX and EPID measurements and phantom position is fixed for all measurements.

The IMatriXX device uses 1020 vented ion chambers, arranged in grid of $32 \times 32$ array, with active area of $24.4 \times 24.4 \mathrm{~cm}^{2}$. The lateral spacing between two ion chambers is 7.62 $\mathrm{mm}$, more details specifications are tabulated in Table 1.

All portal images were taken with a flat panel imager a-Si 1000 (Varian Medical Systems, Palo Alto, USA). It has a $30 \times 40 \mathrm{~cm}^{2}$ detection area with $768 \times 1024$ pixels, phosphor screen, $1.0 \mathrm{~mm} \mathrm{Cu}$ build-up layer and hydrogenated aSi:H photodiode array [15]. The special resolution of EPID is $0.391 \mathrm{~mm}$.

The conversion of PDI pixel values to the dose in Gray (Gy), at the plane of reconstruction and its calibration procedure are described in earlier studies [16-17].

The TPS reconstructs the dose in the mid plane of medium i.e. the phantom, and only dosimetry phantom are used in this study. At all gantry angle, mid-plane is at the isocenter, normal the beam axis. Within sensitive matrix at mid plane, the pixel values are used to convert absolute dose images. This sensitivity matrix is used to account the variation in the response of pixel values over the complete active area of EPID panel.

The verification plan created in TPS were compared with the planned 2D dose distribution in ' $\mathrm{X}$ ' and ' $\mathrm{Y}$ ' plane. EPID dose images were compared with the verification plan created in TPS in two dimensions at the plane intersecting the isocenter, perpendicular to the beam. For each plan, the plane of measurement corresponds to the reconstruction plane for EPID. Dose images reconstructed at mid plane for each field correspond to all segment dose image. In comparison with gamma image, $3 \% / 3 \mathrm{~mm}$ criteria was performed with absolute dose profiles in both planes.

Comparing gamma images was done with $3 \% / 3 \mathrm{~mm}$ criteria, and absolute dose profiles. Plans were considered acceptable if, for verification field, $\gamma_{\mathrm{avg}}=0.50, \gamma_{\max }=3.5$, and area gamma $<1>95 \%$ as tolerance limits and IMatriXX correlation $>95 \%$. The combination of these above parameters provides detailed and informative summary of the overall agreement between planned and measured 2D dose distributions for these 122 patient plans. If we

Table 1: The detailed specifications of Electronic Portal Imaging Device (EPID) and IMatriXX.

\begin{tabular}{cccc} 
Particulars /Dosimetry Devices & \multicolumn{2}{c}{ EPID } & IMatriXX \\
\hline Detector material & a-Si 1000 & a-Si 1200 & Ion Chamber Array \\
\hline Irradiated area $\left(\mathrm{cm}^{2}\right)$ & $30 \times 40$ & $43 \times 43$ & $30 \times 30$ \\
\hline Active area $\left(\mathrm{cm}^{2}\right)$ & $30 \times 40$ & $40 \times 40$ & $24.4 \times 24.4$ \\
\hline Resolution / Pixel size $(\mathrm{mm})$ & 0.391 & 0.336 & 7.62 \\
\hline Active dosimetry matrix & $768 \times 1024$ & $1190 \times 1190$ & 1020 lon Chambers
\end{tabular}

a-Si: Amorphous Silicon 
choose different $\gamma$ criteria, other than $3 \% / 3$ $\mathrm{mm}$, rescaling of these values would be done as per new criteria selected.

\section{Method of Dose Comparison}

The portal dosimetry software was used for the evaluation of dose distribution which is one of workspace ECLIPSE TPS version 11.0, and IMatriXX - OmniPro version 1.7 IBA Dosimetry. With the software, we can compare obtained dose difference values with EPID, IMatriXX, and values obtained from the verification plan created from treatment planning system. The evaluation is based on the dose difference values at a point and in ' $\mathrm{X}$ ' and ' $\mathrm{Y}$ ' profiles 2D distributions. Comparison is mainly based on the use of dose difference images, correlation and the gamma evaluation criteria as described in earlier studies [18-19].

For these evaluations, region of interest (ROI) is drawn for each field and used the criteria of the combination of scalar parameters. The analysis and quantification performed within the ROI i.e. IMatriXX correlation, average gamma $\left(\gamma_{\text {avg }}\right)$, area gamma $<1$ i.e. percentage of points with $\gamma<1$ and maximum $\gamma\left(\gamma_{\max }\right)$ are studied and recorded [20-21].

In $2 \mathrm{D}$ evaluations, $3 \%$ global dose difference gamma criteria and $3 \mathrm{~mm}$ distance to agreement (DTA) were chosen, relative to maximum field dose. We used these criteria for re- sults of some test cases performed using $2 \% /$ $2 \mathrm{~mm}$ criteria [22-23] and extended to $3 \% / 3$ $\mathrm{mm}$ for the clinical practice. The uncertainty can be due to calibration of the EPID and IMatriXX, reproducibility of measurements, resolution of dosimetry devices used in measurement and TPS calculation accuracy.

\section{Results}

Total 122 patients with $\mathrm{H} \& \mathrm{~N}$ cancer were selected for treatment and IMRT treatment plan were verified using EPID and IMatriXX. Out of these 122 patients, 88 were males and 34 females. The head and neck site were subdivided in to five other sub-sites such as oral cavity, pharynx, larynx, paranasal sinus and other (parotid, ear etc.), as shown in Figure 1.

Maximum number of patients are from age group of 61- 70 years old, and maximum dose, planned for treatment is of 74 Gy for carcinoma of pharynx as in Figure 2. IMRT treatment plans for $122 \mathrm{H} \& \mathrm{~N}$ cancer patients were verified at the isocenter plane using aSi-1000 EPID measurements and IMatriXX measurement and analyzed using software ECLIPSE and OmniPro, respectively.

When we observe the values for cancer of Pharynx, $98.425 \%$ of points are seen in $3 \%$ and $3 \mathrm{~mm}$ criteria i.e. area gamma $<1$ equals 98.425\%, maximum gamma $\gamma_{\max }$ equals 1.925 , and average gamma $\gamma_{\text {avg }}$ equals 0.183 point to-

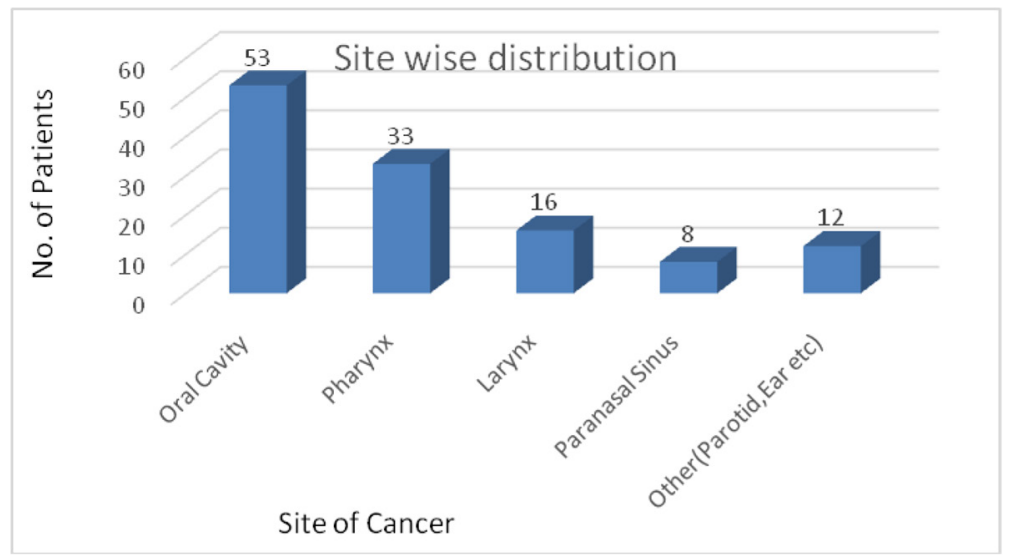

Figure 1: Site wise distribution of patients with maximum patients in the category of oral cavity in this rural setup. 


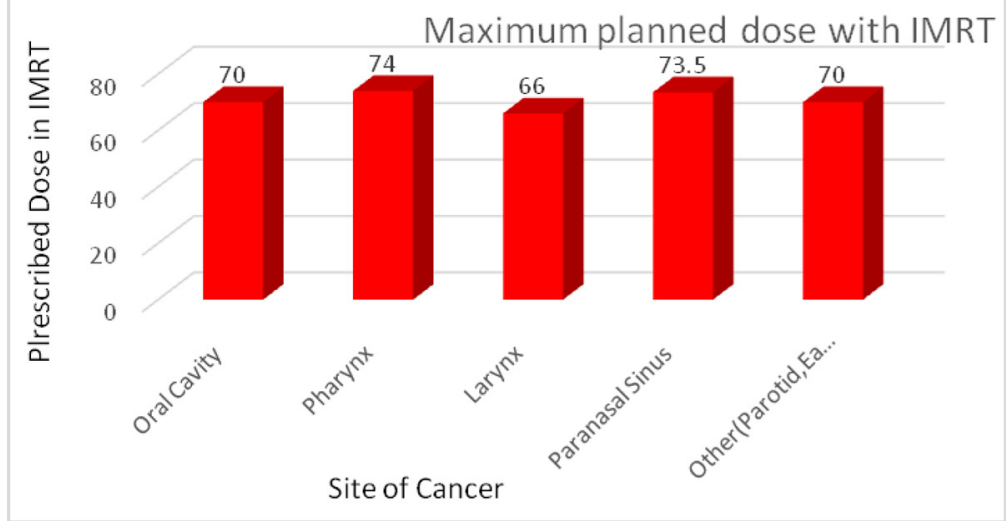

Figure 2: Maximum planned dose with Intensity Modulated Radiotherapy (IMRT) treatment technique. The maximum dose, planned for Pharynx (74 Gy) and minimum dose was for Larynx (66 Gy).

ward improved overall agreement with IMatriXX correlation of $99.0 \%$ as seen in Table 2 .

2D dose distributions from EPID and IMatriXX for total 122 patient plans were verified. With the applied criteria of $3 \% / 3 \mathrm{~mm}$ for all points and image sets, these planned and measured distributions agreed well within tolerance with $\gamma_{\mathrm{avg}}=0.192$ (0.048 SD), $\gamma_{\max }=2.19$ and area gamma $<1=98.18 \%$ as recorded. These values can be set as baseline values for this center during patient specific quality assurance. It is observed that as the value of area gamma $<1$ decreases, the value of $\gamma_{\text {avg }}$ and $\gamma_{\max }$ increases, showing that there is poor agreement between planned and measured 2D dose distribution with decreased IMatriXX correlation.

Some over response is observed in the doses measured for various field sizes at EPID and IMatriXX. Spatial resolution has a great impact on the response for comparing dose distributions in steep dose gradient area.

The Figure 3 shows that both the EPID and IMatriXX correlation can be compared in clinical setting for head and neck cases. Comparisons of gamma criteria 3\% / $3 \mathrm{~mm}$ and dose profiles (absolute values) are done, as seen in Figure 4.

Disagreement is observed in 3 plans out of the 122 verification plans. These 3 plans fail in high dose gradient area, and errors exceeded the acceptance criteria. The IMatriXX correlation and area gamma $<1$ are found to be $81.5 \%, 91.6 \%$ and $87.2 \%$ which are less than acceptable limits of $95.0 \%$. The IMatriXX slightly overestimates the gamma correlation

Table 2: Details of the gamma analysis with acceptable criteria and the actual analysis.

\begin{tabular}{ccccccc}
$\begin{array}{c}\text { Site / Diag- } \\
\text { nosis }\end{array}$ & $\begin{array}{c}\text { No. of } \\
\text { Patients }\end{array}$ & $\begin{array}{c}\text { IMatriXX- } \\
\text { Correlation }\end{array}$ & $\begin{array}{c}\text { IMatriXX- } \\
\text { Histogram }\end{array}$ & $\begin{array}{c}\text { Area Gamma } \\
\mathbf{< 1} \text { (Tol=95\%) }\end{array}$ & $\begin{array}{c}\text { Max Gamma } \\
\text { (Tol=3.50) }\end{array}$ & $\begin{array}{c}\text { Avg Gamma } \\
\text { (Tol = 0.5) }\end{array}$ \\
\hline Oral Cavity & 53 & 0.992 & 92.420 & 98.108 & 2.323 & 0.197 \\
\hline Pharynx & 33 & 0.990 & 92.922 & 98.425 & 1.925 & 0.183 \\
\hline Larynx & 16 & 0.986 & 90.193 & 98.067 & 2.143 & 0.190 \\
\hline Paranasal Sinus & 8 & 0.983 & 88.005 & 97.713 & 2.605 & 0.208 \\
\hline $\begin{array}{c}\text { Other (Parotid, } \\
\text { Ear etc) }\end{array}$ & 12 & 0.991 & 95.318 & 98.317 & 2.128 & 0.197
\end{tabular}


More M., Jain V., Gurjar O. P.

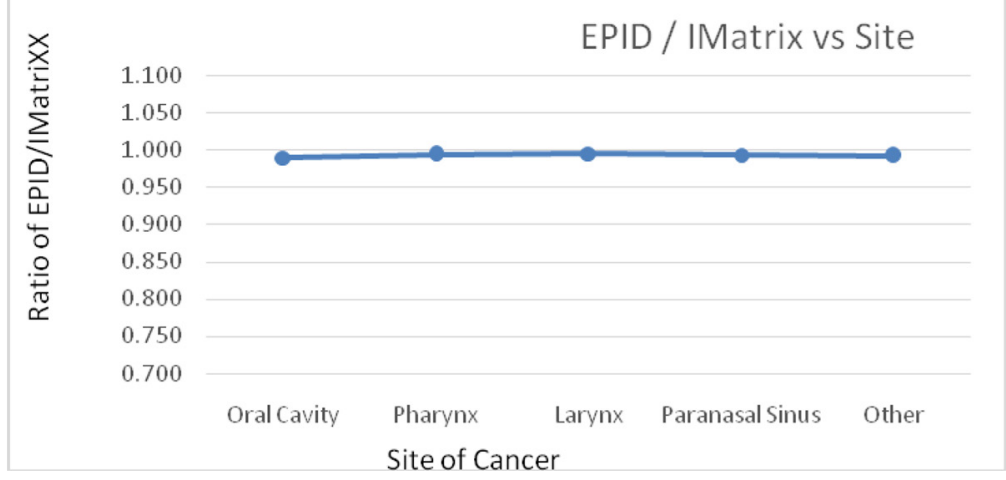

Figure 3: Ratio of Electronic Portal Imaging Device (EPID) / IMatriXX versus site, reflecting that both the methods are equivalent for the plan comparison in a clinical setting.

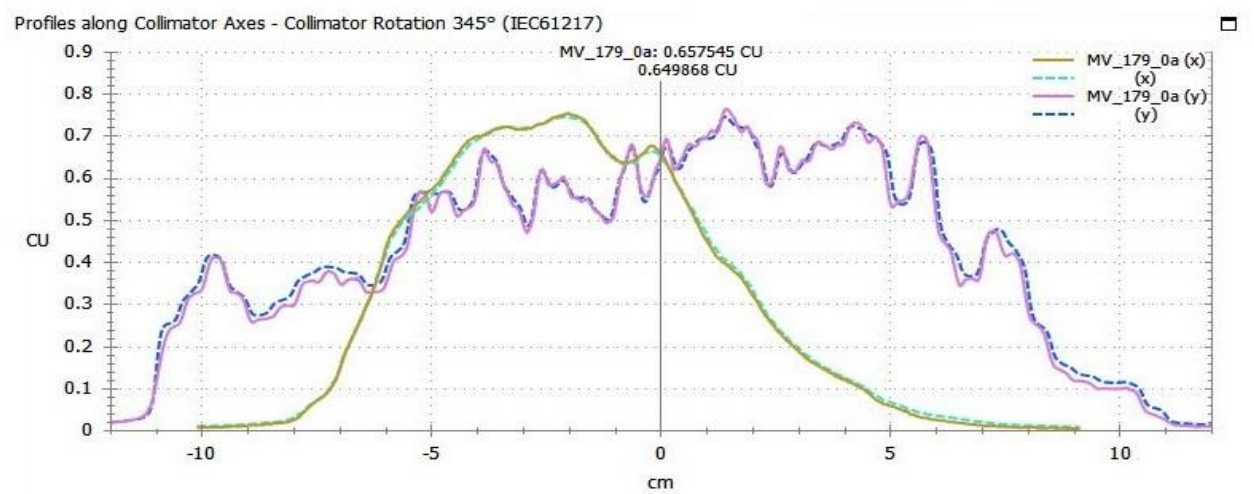

\begin{tabular}{|l|l|l|l|l|}
\hline Gamma (3\% / 3 mm) & Value & Tol. & Abs.Dose Difference & Value \\
\hline Area Gamma $<1$ & $99.9 \%$ & $95.0 \%$ & Max. Dose Difference & $0.12 \mathrm{CU}$ \\
\hline Maximum Gamma & 1.77 & 3.50 & Avg. Dose Difference & $0.01 \mathrm{CU}$ \\
\hline Average Gamma & 0.20 & 0.50 & Area Dose Difference $>0.50 \mathrm{CU}$ & $0.0 \%$ \\
\hline Area Gamma $>0.8$ & $0.4 \%$ & & Area Dose Difference $>0.80 \mathrm{CU}$ & $0.0 \%$ \\
\hline Area gamma $>1.2$ & $0.0 \%$ & & RESULT & PASSED \\
\hline
\end{tabular}

Figure 4: Comparison of predicted dose and measured dose with portal dosimetry workspace in ECLIPSE treatment planning system.

as it has resolution of $7.62 \mathrm{~mm}$ and interpolates values between missing measurement points in dose image (TG-218) [24].

The line profiles of these 3 plans with disagreement revealed that there may exist high dose gradient regions, leading to lower values of gamma passing. The variation of about $3.4 \%-13.5 \%$ were observed which is lower than the reference tolerance value of $95 \%$. For these cases, the treatment plan was performed again with modified plan parameters, reducing high dose gradient regions in the dose distri- bution and planned and measured dose distributions shows overall improved agreement.

\section{Discussion}

With the advanced treatment technique like IMRT, the requirement for $\mathrm{QA}$ in the radiotherapy clinic has rapidly increased than earlier conventional technique. EPID dosimetry has been focused in the majority of studies and reported on various verification methods, the dose response characteristics and various algorithms applied. Purpose of EPID dosim- 
etry method in the 'clinical application' is to directly influence accuracy in the radiation dose delivery to cancer patient with the intent to improve correctness in treatment, resulting in benefit to the patients.

Pre-treatment evaluation for large patient groups has been reported by other authors using film dosimetry not EPID. Stock et al., [25] reported as average gamma $\gamma_{\mathrm{avg}}=0.45 \pm 0.1$ when using $3 \%$ global dose $3 \mathrm{~mm}$ criteria. The results of our study are comparable with the study of Stock et al., [25] proving the sensitivity of gamma criteria for IMRT plan verification. The results of this study using 3\% global dose $3 \mathrm{~mm}$ criteria for evaluation shows the average gamma is $0.214 \pm 0.024$ with EPID, in carcinoma head and neck patient. These results are comparable to earlier published studies.

Chang et al., [26] compared IMRT prostate fields' of 25 patients' dose profiles and dose at the central axis using portal imager and TPS designed dose distributions. The measured agreement was within $2 \%$, nearby the errors related to image acquisition. It was accomplished that the portal dosimetry system is an efficient authentication tool for IMRT pretreatment plan verification.

The aim of the study is to reveal the sensitivity and correctness of portal dosimetry system as a PSQA means. We establish EPID and IMatriXX dosimetry to be more responsive, sensitive and competent, providing the similar information and high accuracy in both EPID \& IMatriXX dosimetry systems.

The time taken for dose measurement varies based on methods, depending upon the equipment and software used. It is observed that acquisition of digital portal dose image is all the time more rapid than the other dose images measurement technique.

\section{Conclusion}

We revealed how portal dose back-projection dosimetry technique is able to verify clinical IMRT plans for head and neck cancer patients prior to actual treatment. It is concluded from the results of 122 patients that the portal imager can substitute other dosimetry systems used in pretreatment plan verification and PSQA. EPID has advantage over other dosimetry devices like IMatriXX because of its high resolution of $0.391 \mathrm{~mm}$.

There exists close agreement between dose measured using ion-chamber at isocenter plane and portal dose measurement for the pretreatment plan verification, thus portal dosimetry can replace routine ion-chamber measurements.

The results of portal dosimetry for 122 patients are used to precisely confirm the planned distribution in 2D plane and also extended to setup the baseline values for our center.

EPID dosimetry also called as 'Portal dosimetry' is an efficient, precise and sensitive dosimetry tool for IMRT plan verification and the basis of pre-treatment quality assurance protocol.

\section{Acknowledgment}

The authors are thankful to the physicists for measurement and computation of patient treatment plans on EPID and IMatriXX phantom.

\section{Conflict of Interest}

None

\section{References}

1. El-Mohri Y, Antonuk LE, Yorkston J, Jee KW, et al. Relative dosimetry using active matrix flat-panel imager (AMFPI) technology. Med Phys. 1999;26(8):1530-41. doi: 10.1118/1.598649. PubMed PMID: 10501053.

2. McCurdy BM, Luchka K, Pistorius S. Dosimetric investigation and portal dose image prediction using an amorphous silicon electronic portal imaging device. Med Phys. 2001;28(6):911-24. doi: 10.1118/1.1374244. PubMed PMID: 11439488.

3. Grein EE, Lee R, Luchka K. An investigation of a new amorphous silicon electronic portal imaging device for transit dosimetry. Med Phys. 2002;29(10):2262-8. doi: 10.1118/1.1508108. PubMed PMID: 12408300.

4. Greer PB, Popescu CC. Dosimetric properties of an amorphous silicon electronic portal imaging device for verification of dynamic intensity modulated radiation therapy. Med Phys. 2003;30(7):1618-27. doi: 
10.1118/1.1582469. PubMed PMID: 12906179.

5. Warkentin B, Steciw S, Rathee S, Fallone BG. Dosimetric IMRT verification with a flat-panel EPID. Med Phys. 2003;30(12):3143-55. doi: 10.1118/1.1625440. PubMed PMID: 14713081.

6. McDermott LN, Louwe RJ, Sonke JJ, Van Herk MB, Mijnheer BJ. Dose-response and ghosting effects of an amorphous silicon electronic portal imaging device. Med Phys. 2004;31(2):285-95. doi: 10.1118/1.1637969. PubMed PMID: 15000614.

7. Louwe RJ, McDermott LN, Sonke JJ, et al. The long-term stability of amorphous silicon flat panel imaging devices for dosimetry purposes. Med Phys. 2004;31(11):2989-95. doi: 10.1118/1.1803751. PubMed PMID: 15587651.

8. Reiner BI, Siegel EL, Siddiqui K. Evolution of the digital revolution: a radiologist perspective. J Digit Imaging. 2003;16(4):324-30. doi: 10.1007/s10278003-1743-y. PubMed PMID: 14747936. PubMed PMCID: PMC3044070.

9. Georg D, Kroupa B, Winkler P, Pötter R. Normalized sensitometric curves for the verification of hybrid IMRT treatment plans with multiple energies. Med Phys. 2003;30(6):1142-50. doi: 10.1118/1.1576951. PubMed PMID: 12852539.

10. Stock M, Kroupa B, Georg D. Interpretation and evaluation of the gamma index and the gamma index angle for the verification of IMRT hybrid plans. Phys Med Biol. 2005;50(3):399-411. doi: 10.1088/00319155/50/3/001. PubMed PMID: 15773719.

11. Jursinic PA, Nelms BE. A 2-D diode array and analysis software for verification of intensity modulated radiation therapy delivery. Med Phys. 2003;30(5):870-9. doi: 10.1118/1.1567831. PubMed PMID: 12772995.

12. Létourneau D, Gulam $M$, Yan D, Oldham M, Wong JW. Evaluation of a 2D diode array for IMRT quality assurance. Radiother Oncol. 2004;70(2):199-206. doi: 10.1016/j.radonc.2003.10.014. PubMed PMID: 15028408.

13. Wiezorek T, Banz N, Schwedas M, et al. Dosimetric quality assurance for intensity-modulated radiotherapy feasibility study for a filmless approach. Strahlenther Onkol. 2005;181(7):468-74. doi: 10.1007/ s00066-005-1381-z. PubMed PMID: 15995841.

14. Childress NL, Bloch C, White RA, Salehpour M, Rosen II. Detection of IMRT delivery errors using a quantitative 2D dosimetric verification system. Med Phys. 2005;32(1):153-62. doi: 10.1118/1.1829171. PubMed PMID: 15719966.

15. Steciw S, Warkentin B, Rathee S, Fallone BG. Three-dimensional IMRT verification with a flatpanel EPID. Med Phys. 2005;32(2):600-12. doi: 10.1118/1.1843471. PubMed PMID: 15789607.

16. Wendling M, Louwe RJ, McDermott LN, Sonke JJ, et al. Accurate two-dimensional IMRT verification us- ing a back-projection EPID dosimetry method. Med Phys. 2006;33(2):259-73. doi: 10.1118/1.2147744. PubMed PMID: 16532930.

17. Low DA, Harms WB, Mutic S, Purdy JA. A technique for the quantitative evaluation of dose distributions. Med Phys. 1998;25(5):656-61. doi: 10.1118/1.598248. PubMed PMID: 9608475.

18. Kim YL, Chung JB, Kim JS, Lee JW, Choi KS. Comparison of the performance between portal dosimetry and a commercial two-dimensional array system on pretreatment quality assurance for volumetricmodulated arc and intensity-modulated radiation therapy. Journal of the Korean Physical Society. 2014;64(8):1207-12. doi: 10.3938/jkps.64.1207.

19. Winkler P, Zurl B, Guss H, Kindl P, Stuecklschweiger G. Performance analysis of a film dosimetric quality assurance procedure for IMRT with regard to the employment of quantitative evaluation methods. Phys Med Biol. 2005;50(4):643-54. doi: 10.1088/00319155/50/4/006. PubMed PMID: 15773625.

20. Van Zijtveld M, Dirkx ML, De Boer HC, Heijmen BJ. Dosimetric pre-treatment verification of IMRT using an EPID; clinical experience. Radiotherapy and Oncology. 2006;81(2):168-75. doi: 10.1016/j.radonc.2006.09.008.

21. Budgell GJ, Perrin BA, Mott JH, Fairfoul J, Mackay RI. Quantitative analysis of patient-specific dosimetric IMRT verification. Phys Med Biol. 2005;50(1):10319. doi: $10.1088 / 0031-9155 / 50 / 1 / 009$. PubMed PMID: 15715426.

22. Partridge M, Symonds-Tayler JR, Evans PM. IMRT verification with a camera-based electronic portal imaging system. Phys Med Biol. 2000;45(12):N183-96. doi: 10.1088/0031-9155/45/12/402. PubMed PMID: 11131208.

23. Louwe RJ, Damen EM, Van Herk M, et al. Threedimensional dose reconstruction of breast cancer treatment using portal imaging. Med Phys. 2003;30(9):2376-89. doi: 10.1118/1.1589496. PubMed PMID: 14528960.

24. Miften M, Olch A, Mihailidis D, Moran J, et al. Tolerance limits and methodologies for IMRT measurementbased verification QA: Recommendations of AAPM Task Group No. 218. Med Phys. 2018;45(4):e53-83. doi: 10.1002/mp.12810. PubMed PMID: 29443390.

25. Herzen J, Todorovic M, Cermers F, et al. Dosimetric evaluation of a $2 \mathrm{D}$ pixel ionization chamber for implementation in clinical routine. Phys Med Biol. 2007;52(4):1197-208. doi: 10.1088/00319155/52/4/023.

26. Chang J, Ling CC. Using the frame averaging of aS500 EPID for IMRT verification. J Appl Clin Med Phys. 2003;4(4):287-99. doi: 10.1120/jacmp. v4i4.2499. PubMed PMID: 14604418. PubMed PMCID: PMC5724460. 